BMC

Genomics

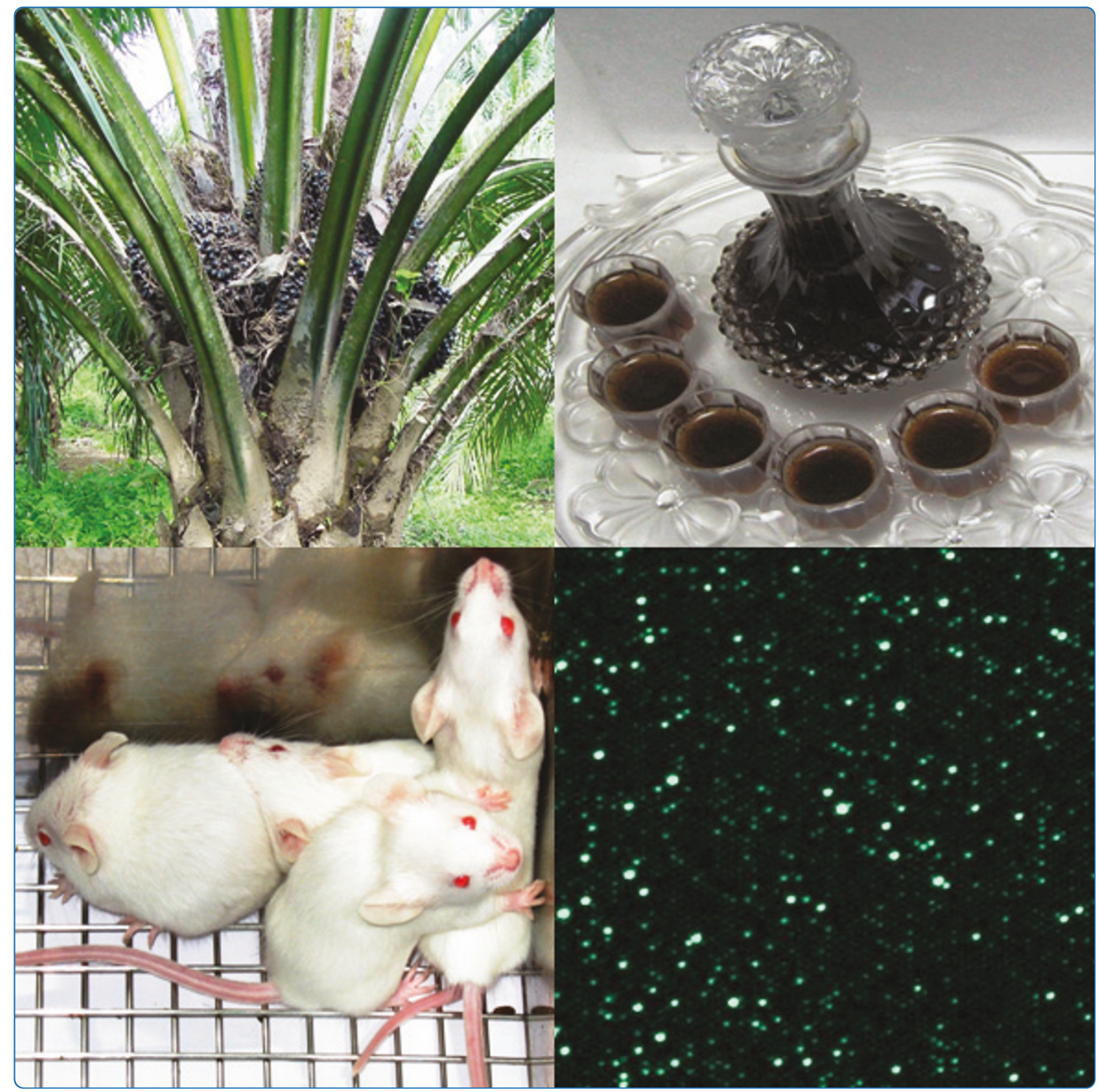

Differential transcriptomic profiles effected by oil palm phenolics indicate novel health outcomes

Leow et al.

C Biomed Central

Leow et al. BMC Genomics 2011, 12:432

http://www.biomedcentral.com/1471-2164/12/432 (25 August 2011) 


\title{
Differential transcriptomic profiles effected by oil palm phenolics indicate novel health outcomes
}

\author{
Soon-Sen Leow ${ }^{1}$, Shamala Devi Sekaran², Kalyana Sundram³ ${ }^{3}$ YewAi Tan ${ }^{1}$ and Ravigadevi Sambanthamurthi ${ }^{*}$
}

\begin{abstract}
Background: Plant phenolics are important nutritional antioxidants which could aid in overcoming chronic diseases such as cardiovascular disease and cancer, two leading causes of death in the world. The oil palm (Elaeis guineensis) is a rich source of water-soluble phenolics which have high antioxidant activities. This study aimed to identify the in vivo effects and molecular mechanisms involved in the biological activities of oil palm phenolics (OPP) during healthy states via microarray gene expression profiling, using mice supplemented with a normal diet as biological models.
\end{abstract}

Results: Having confirmed via histology, haematology and clinical biochemistry analyses that OPP is not toxic to mice, we further explored the gene expression changes caused by OPP through statistical and functional analyses using Illumina microarrays. OPP showed numerous biological activities in three major organs of mice, the liver, spleen and heart. In livers of mice given OPP, four lipid catabolism genes were up-regulated while five cholesterol biosynthesis genes were down-regulated, suggesting that OPP may play a role in reducing cardiovascular disease. OPP also up-regulated eighteen blood coagulation genes in spleens of mice. OPP elicited gene expression changes similar to the effects of caloric restriction in the hearts of mice supplemented with OPP. Microarray gene expression fold changes for six target genes in the three major organs tested were validated with real-time quantitative reverse transcription-polymerase chain reaction (qRT-PCR), and the correlation of fold changes obtained with these two techniques was high $\left(R^{2}=0.9653\right)$.

Conclusions: OPP showed non-toxicity and various pleiotropic effects in mice. This study implies the potential application of OPP as a valuable source of wellness nutraceuticals, and further suggests the molecular mechanisms as to how dietary phenolics work in vivo.

\section{Background}

The continuous improvement in the quality of life in recent years, together with the advancement of science and technology, has created a more health-conscious society. Data from large population studies suggest that lifestyle factors, such as sedentary lifestyle, dietary intake and adiposity are responsible for $70 \%$ of chronic diseases and are a major contributor to reduced longevity [1]. Thus, society now realises the importance of physical activity and dietary intervention towards the prevention of chronic diseases and ageing. Chronic diseases such as cardiovascular disease and cancer are by far the leading

\footnotetext{
*Correspondence: raviga@mpob.gov.my

'Malaysian Palm Oil Board, No. 6, Persiaran Institusi, Bandar Baru Bangi,

43000 Kajang, Selangor, Malaysia

Full list of author information is available at the end of the article
}

cause of mortality in the world, representing $60 \%$ of all deaths [2].

Prooxidants such as reactive oxygen species (ROS) play important roles in triggering chronic diseases. For example, the hydroxyl radical causes lipid peroxidation, modification of DNA bases or protein damage and in turn, leads to tissue damage, chronic diseases and ageing [3]. In addition to oxidising macromolecules such as DNA in the body, prooxidants have been found to regulate gene expression, of which two well-defined transcription factors, nuclear factor kappa-B and activator protein-1 are affected, causing inflammation [4]. Inflammation thus links oxidative stress with chronic diseases.

Many studies conducted recently have shown that diets containing high amounts of phytochemicals can provide protection against these prooxidant-induced diseases, due to their high antioxidant activities. 
Antioxidants preclude prooxidant-induced tissue damage by preventing the formation of these prooxidants, by scavenging them or by promoting their decomposition [3]. Antioxidants are also found to be caloric restriction mimetics, the only intervention known to lengthen the median lifespan of animals [5]. Among the different groups of naturally occurring antioxidants from plants, carotenoids and phenolics are perhaps the two most important [6]. Phenolics comprise an aromatic ring, and are important antioxidants because of their high redox potential, which allows them to act as reducing agents, hydrogen donors, singlet oxygen quenchers and metal chelators [6]. Around 8000 phenolic compounds have been identified thus far, and they have been classified into different categories based on their basic structures [7]. Phenolics possess many therapeutic properties. For example, flavonoids have been shown to have antioxidant, neuroprotective, cardioprotective, lipid-lowering, anti-hepatotoxic, anti-allergic, anti-cancer, anti-inflammatory, anti-thrombotic and anti-microbial activities [8].

Besides their antioxidant properties, phytochemicals such as phenolics are also known to influence gene expression [9]. Microarray analysis has thus been suggested as a tool to identify modulations of multiple gene networks by antioxidant micronutrients [10], as the expression profiles of thousands of genes can be measured in a single experiment [11]. As dietary intervention generally results in small effects on a big number of genes compared to pharmaceutical intervention which targets a specific biomarker, microarrays can therefore detect a combined effect of several genes belonging to a similar biological pathway [12]. Indeed, multi-component botanical therapeutics such as phenolics may become particularly valuable in the long-term prevention and treatment of complex diseases requiring extended administration and pleiotropic action [13]. In studies on plant phenolics, microarray studies have been carried out to test the biological effects of flavonoids in cultured cell lines in vitro [14,15] as well as in animals in vivo [12].

The oil palm (Elaeis guineensis) contains an excellent repertoire of antioxidant phytochemicals. Palm fruit oil contains many lipid-soluble antioxidants, such as carotenoids (precursors of vitamin A), as well as tocopherols and tocotrienols (isomers of vitamin E) [16-18]. Another relatively new antioxidant phytochemical from the oil palm fruit is the water-soluble phenolic acid-rich complex, which is recovered from the oil palm vegetation liquor through a series of centrifugation and membrane filtration steps [19]. These oil palm phenolics (OPP) consist mainly of phenolic acids, including three caffeoylshikimic acid isomers, protocatechuic acid and $p$ hydroxybenzoic acid [20,21]. OPP has been shown to display antioxidant properties and confer positive outcomes on degenerative diseases in various animal models without evidence of causing toxicity [21-23]. The utilisation of OPP in the nutraceutical market is however, subject to understanding the molecular mechanisms involved in bringing about the positive benefits observed, whether in healthy or diseased states. The aim of this study was thus to identify the in vivo biological effects and molecular mechanisms of these compounds during healthy states in three major organs (liver, spleen and heart) of mice on a normal diet, via microarray gene expression profiling.

\section{Results}

Weight gain delay and non-toxicity in mice supplemented with OPP

We first confirmed that OPP was not toxic to mice via physiology, histology, haematology and clinical biochemistry analyses. The body weights of mice steadily increased every week throughout the six weeks of feeding, with mice given OPP showing a delayed weight gain (Figure 1A). OPP did not significantly affect organ weights (Figure 1B), fluid intake (Figures $1 \mathrm{C}$ and 1D), food intake (Figures $1 \mathrm{E}$ and $1 \mathrm{~F}$ ), faecal output (Figures $1 \mathrm{G}$ and $1 \mathrm{H}$ ), organ histology (Figure 2), as well as haematology and clinical biochemistry (Table 1) parameters.

\section{Overview of gene expression changes in the liver, spleen and heart}

Illumina microarrays were used to study the effects of OPP in the livers, spleens and hearts of mice given a normal diet. OPP significantly changed 250 genes in the liver (196 genes up-regulated and 54 genes down-regulated), 142 genes in the spleen (100 genes up-regulated and 42 genes down-regulated), and 475 genes in the heart (196 genes up-regulated and 279 genes downregulated). The number of genes significantly changed by OPP was thus highest in the heart, followed by the liver and the spleen. The lists of genes significantly changed by OPP in these organs, together with their fold changes, are supplemented in Additional File 1.

The effects of OPP on the organs tested were quite subtle. The majority of the differentially expressed genes rarely exceeded two fold change in expression, with the exception of genes considered turned on (significantly detected only in the treatment group) or off (significantly detected only in the control group). The large fold changes observed in this particular group of genes (turned on or off) are arbitrary values as values of 10 were assigned when negative expression values were observed after normalisation. These results indicate that OPP can act as regulators of gene expression, turning on and silencing genes. In addition, the low fold changes caused by OPP indicate that they do not have drastic 

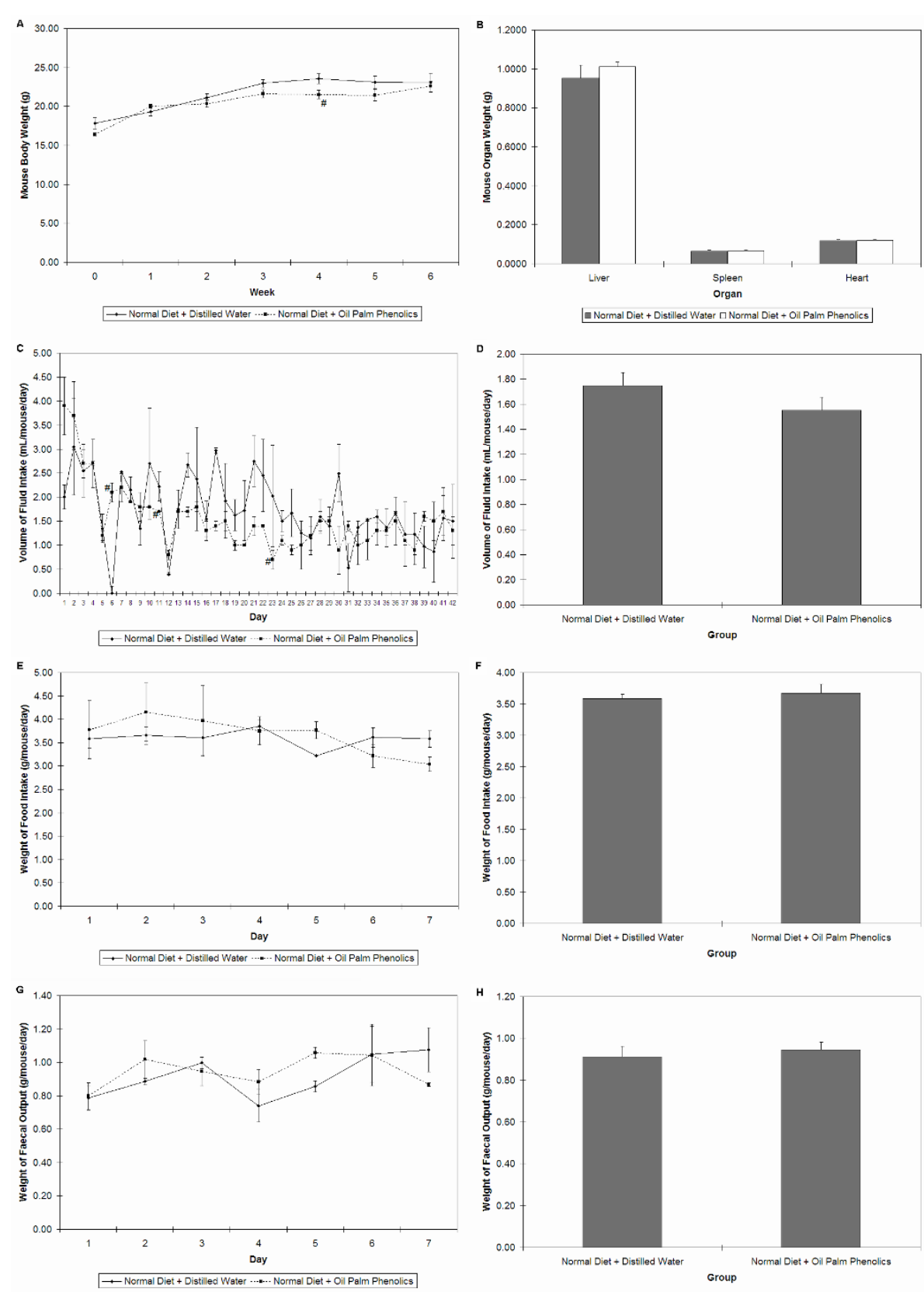

Figure 1 Physiology parameters of mice. (A) Body weights; $n=10$. (B) Organ weights; $n=10$. (C) Timeline of fluid intake; $n=2$ cages (of 5 mice per cage). (D) Average daily fluid intake; $n=42$ days. (E) Timeline of food intake, $n=2$ cages (of 5 mice per cage). (F) Average daily food intake between week two to week three; $n=7$ days. (G) Timeline of faecal output, $n=2$ cages (of 5 mice per cage). (H) Average daily faecal output between week two to week three; $n=7$ days. \# $P<0.05$ vs. Normal Diet + Distilled Water. Error bars indicate s.e.m.

effects on gene expression in general, and may be used as dietary supplements. Such supplements should not bring too drastic a change in the system of an organism, but rather act as an antioxidant buffer to cushion the effects of other environmental influences such as prooxidant production.

An example of the two-way hierarchical clustering analysis carried out on significantly changed genes in the liver is given in Additional File 2. The replicates of each condition were clustered to one another, indicating robustness of the filtering criteria used. In order to get an overview of the significant functions affected by OPP, we then subjected the microarray data to functional enrichment analysis using the GenMAPP [24] and MAPPFinder [25] softwares (University of California at San Francisco, San Francisco, CA). The lists of these 


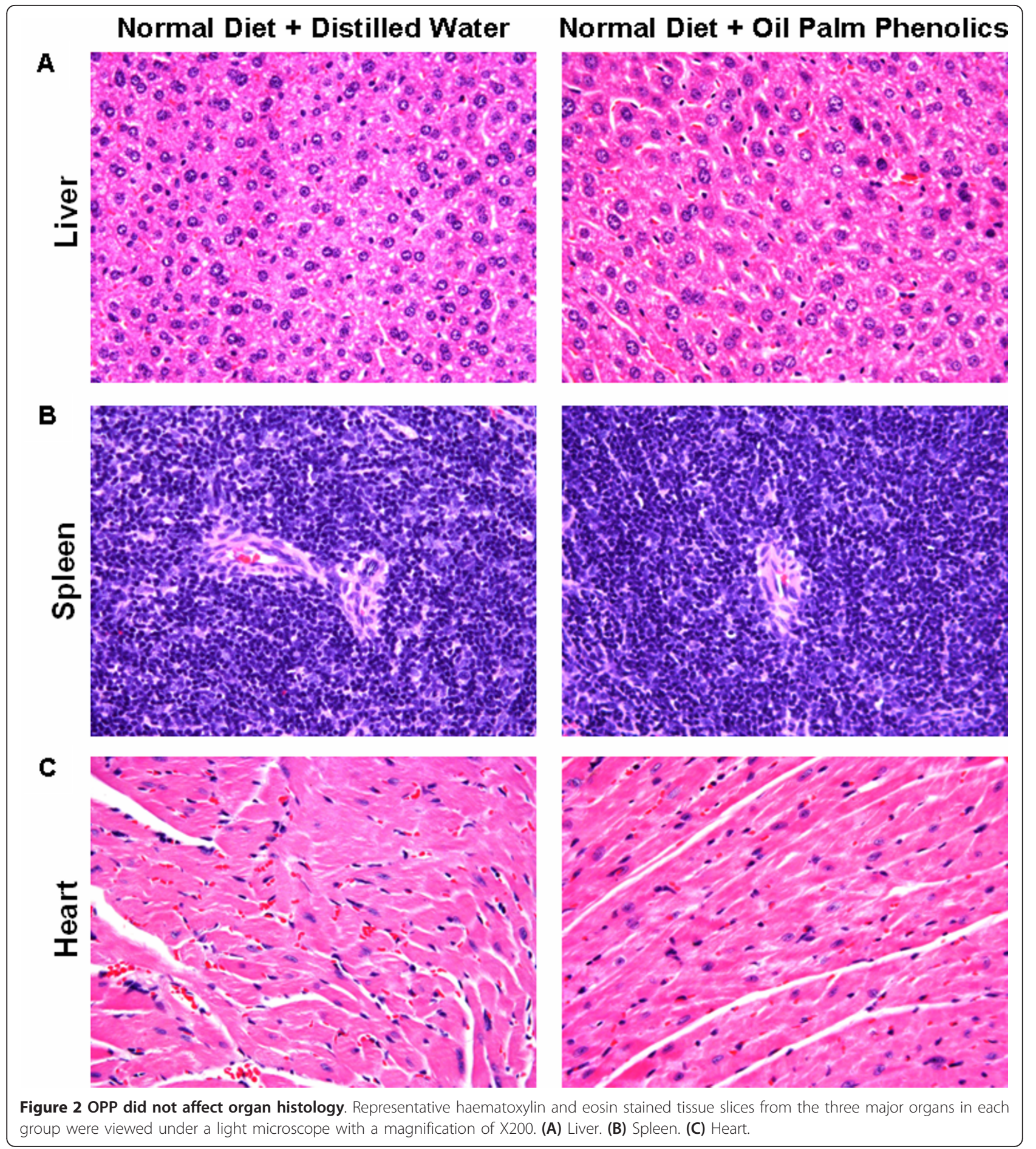

significantly changed functions are provided in Additional File 3. By exploring functions which were considered significantly changed, we further selected GenMAPPs which were considered interesting for pathway analysis. Network analysis was also carried out on the differentially expressed genes by using the Ingenuity
Pathways Analysis software (Ingenuity ${ }^{\circledR}$ Systems, Redwood City, CA) [26].

Gene expression changes in the liver

In the GenMAPPs and gene ontologies found significantly changed in the liver, those related to fatty acid 
Table 1 Haematology and clinical biochemistry parameters of mouse blood samples

\begin{tabular}{|c|c|c|}
\hline Test & $\begin{array}{l}\text { Normal Diet }+ \\
\text { Distilled Water }\end{array}$ & $\begin{array}{c}\text { Normal Diet }+ \\
\text { Oil Palm Phenolics }\end{array}$ \\
\hline Haematology & $(n=4)$ & $(n=4)$ \\
\hline Red Blood Cells (X10 $12 / L)$ & $9.93 \pm 0.32$ & $10.15 \pm 0.12$ \\
\hline Haemoglobin $(\mathrm{g} / \mathrm{L})$ & $148 \pm 4$ & $149 \pm 1$ \\
\hline Haematocrit/Packed Cell Volume (L/L) & $0.40 \pm 0.01$ & $0.40 \pm 0.00$ \\
\hline Mean Corpuscular Volume (fL) & $41 \pm 1$ & $40 \pm 0$ \\
\hline Mean Corpuscular Haemoglobin Concentration (g/L) & $369 \pm 6$ & $373 \pm 4$ \\
\hline White Blood Cells (X109/L) & $2.0 \pm 0.6$ & $1.5 \pm 0.3$ \\
\hline Band Neutrophils $\left(X 10^{9} / \mathrm{L}\right)$ & $0.05 \pm 0.01$ & $0.04 \pm 0.01$ \\
\hline Segmented Neutrophils $\left(X 10^{9} / \mathrm{L}\right)$ & $0.48 \pm 0.17$ & $0.35 \pm 0.09$ \\
\hline Lymphocytes $\left(X 10^{9} / \mathrm{L}\right)$ & $1.36 \pm 0.38$ & $1.01 \pm 0.20$ \\
\hline Monocytes $\left(X 10^{9} / L\right)$ & $0.09 \pm 0.02$ & $0.07 \pm 0.02$ \\
\hline Eosinophils $\left(X 10^{9} / \mathrm{L}\right)$ & $0.03 \pm 0.01$ & $0.01 \pm 0.00$ \\
\hline Basophils $\left(X 10^{9} / \mathrm{L}\right)$ & $0.00 \pm 0.00$ & $0.00 \pm 0.00$ \\
\hline Thrombocytes $\left(X 10^{9} / \mathrm{L}\right)$ & $533 \pm 111$ & $621 \pm 103$ \\
\hline Prothrombin (g/L) & $79 \pm 2$ & $80 \pm 1$ \\
\hline Clinical Biochemistry & $(n=8)$ & $(n=7)$ \\
\hline Alanine Aminotransferase $(U / L)$ & $34.4 \pm 3.3$ & $42.5 \pm 6.5$ \\
\hline Aspartate Aminotransferase (U/L) & $175.2 \pm 23.8$ & $240.4 \pm 22.3$ \\
\hline Glucose $(\mathrm{mmol} / \mathrm{L})$ & $6.0 \pm 1.1$ & $6.7 \pm 0.4$ \\
\hline Serum Total Protein $(\mathrm{g} / \mathrm{L})$ & $53.8 \pm 1.8$ & $53.8 \pm 1.1$ \\
\hline Albumin $(g / L)$ & $34.0 \pm 0.9$ & $33.1 \pm 1.3$ \\
\hline Globulin (g/L) & $19.8 \pm 1.1$ & $20.8 \pm 0.8$ \\
\hline Albumin:Globulin & $1.8 \pm 0.1$ & $1.6 \pm 0.1$ \\
\hline Total Cholesterol (mmol/L) & $3.46 \pm 0.13$ & $3.53 \pm 0.19$ \\
\hline Triglycerides $(\mathrm{mmol} / \mathrm{L})$ & $1.05 \pm 0.08$ & $1.04 \pm 0.11$ \\
\hline Low-Density Lipoproteins (mmol/L) & $0.15 \pm 0.02$ & $0.18 \pm 0.03$ \\
\hline High-Density Lipoproteins (mmol/L) & $2.79 \pm 0.11$ & $2.83 \pm 0.17$ \\
\hline
\end{tabular}

Values shown are means \pm s.e.m. Statistical analysis did not show significant differences in all the parameters measured.

beta oxidation (for up-regulated genes) and cholesterol biosynthesis (for down-regulated genes), were predominant. Further analysis on the fatty acid beta oxidation GenMAPP (pathway) revealed the up-regulation of genes such as those encoding long chain acetyl-CoA dehydrogenase (Acadl), short chain acyl-CoA dehydrogenase (Acads) and hydroxyacyl-CoA dehydrogenases (Hadhb, Hadhsc) (Figure 3A). These significantly upregulated genes are highlighted in yellow.

Other functions which were significantly up-regulated by OPP in the liver were complement activation, protein biosynthesis and transport, ion and electron transport as well as translation factors. Up-regulated genes involved in complement activation include $\mathrm{C} 1 \mathrm{r}, \mathrm{C} 1 \mathrm{rl}, \mathrm{C} 1 \mathrm{~s}, \mathrm{C4}, \mathrm{C} 9$ and $C f h l 1$. In addition, genes involved in ion and electron transport such as those encoding cytochromes $\mathrm{P} 450$ involved in phase I metabolism (Cyp1a2, Cyp27a1, Cyp2a12, Cyp2c54, Cyp2d26, Cyp3a11) and NADH dehydrogenase (ubiquinone) Fe-S proteins (Ndufs2, Ndufs3), were up-regulated. Genes involved in phase II metabolism such as those encoding UDP glycosyltransferases (Ugt1a6 and Ugt1a9) and catechol-O-methyltransferase
(Comt), were up-regulated as well. Translation factors involved in protein synthesis (Eef1d, Eef2, Eif3s2, Eif3s8, Eif4a2, Eif5a) were also up-regulated by OPP.

Genes involved in cholesterol biosynthesis on the other hand, such as those encoding lanosterol synthase (Lss), sterol-C4-methyl oxidase-like (Sc4mol), farnesyl diphosphate synthetase ( $F d p s)$, NAD(P) dependent steroid dehydrogenase-like (Nsdhl) and 3-hydroxy-3-methylglutaryl-CoA synthase 1 (Hmgcs1) were down-regulated (Figure 3B). In this pathway, besides those highlighted in blue (genes which were significantly down-regulated), other genes in the pathway had negative fold changes, the most important being Hmgcr (hydroxymethylglutaryl-CoA reductase), a gene targeted by drugs such as statins for lowering cholesterol. The fold change difference of this gene was however, not significant due to the filtering criteria used.

\section{Gene expression changes in the spleen}

A majority of the genes significantly up-regulated in the spleen were part of the blood coagulation network (Figure 4). Some of the up-regulated genes were those 


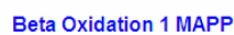

Beta Oxidation Meta MAPP

Beta Oxidation 2 MAPP

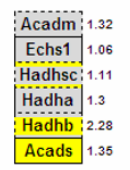

Beta Oxidation 3 MAPP

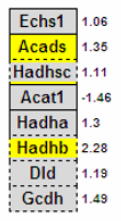

Author: Charles Redfern, NathanSalomonis and Chris Evelo Maintained by: Chris Evelo for NuGO E-mail: mapps@bigcatunimaas.nl
Last modified: Inferred from human Last modified: Inferred from human

http:///Www.bigcat unimaas.nl/mapps/
Copyright $\odot$ Gladstone Institutes and BiGCaT Bioinformatics

\section{Gene Database}

Mm-Std_20060628.gdb

llame: NDL

Gene Value: Hormal Diet. Livers: Fold_Change

Legend: Normal Diet - Livers

$\square$ Up.regulated genes

NNo criteria met

Acadvl 1.02

Hadha 1.3

Acadl :2

Echs1 1.06

Hadhsc:1.11

Lipe 1.04

\begin{tabular}{|c|c|c|c|}
\hline Pnpla2 & -1.28 \\
\hline
\end{tabular}

B

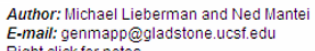
E-mail: genmapp@gladstone.ucsf.ed

Right click for notes
Copyright $\odot 2004$, Gladstone Institutes

Cholesterol Biosynthesis

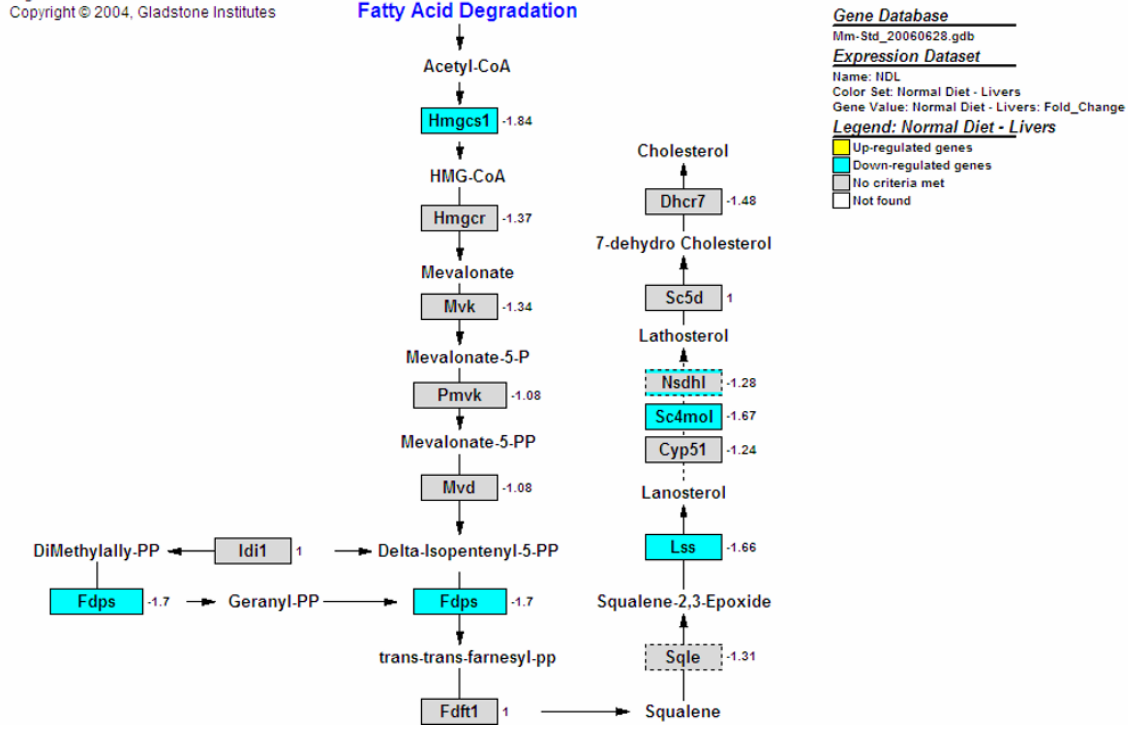

Figure 3 GenMAPPs showing functions and genes significantly changed by OPP in the liver. (A) Genes up-regulated in the liver fatty acid beta oxidation pathway. This GenMAPP represents an overview of three individual fatty acid beta oxidation GenMAPPs, and shows the upregulation of fatty acid beta oxidation genes such as acetyl-CoA dehydrogenase (Acad), acyl-CoA dehydrogenase (Acads) and hydroxyacyl-CoA dehydrogenases (Hadhb, Hadhsc). (B) Genes down-regulated in the liver cholesterol biosynthesis pathway. Note that the fold changes for most of the genes in this GenMAPP were negative, indicating down-regulation, even for genes which were not selected as significantly different based on the selection criteria used. Also note that Hmgcr which encodes for 3-hydroxy-3-methylglutaryl-CoA reductase, an enzyme inhibited by cholesterol-lowering statins, showed a negative fold change as well, although the value was not statistically significant.

encoding Von Willebrand factor homologue ( $V w f), \mathrm{P}-$ selectin (Selp), CD9 antigen (Cd9), integrin alpha $2 \mathrm{~b}$ (Itga 2b), multimerin 1 (Mmrn1), various glycoproteins (Gp1ba, Gp1bb, Gp5, Gp6, Gp9) and thrombin receptors
(F2rl2, F2rl3). These genes are indicated in red in the blood coagulation network.

While a pathway involves the metabolism of biochemical compounds catalysed by enzymes, a network 


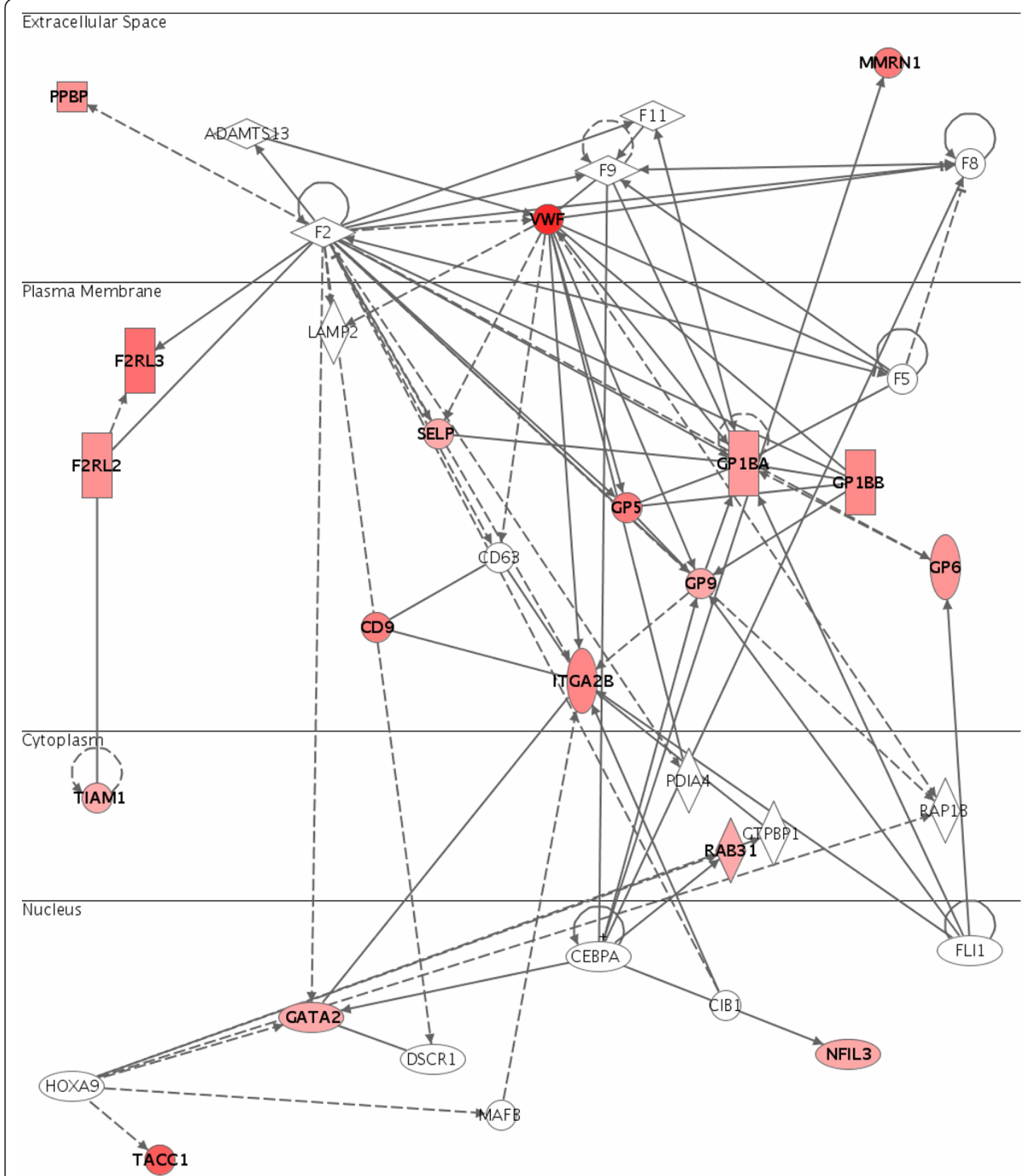

Figure 4 Genes involved in the spleen blood coagulation network which were up-regulated by OPP. The up-regulation of various genes involved in blood coagulation such as those encoding Von Willebrand factor homologue (Vwf), P-selectin (Selp), various glycoproteins (Gp1ba, Gp1bb, Gp5, Gp6, Gp9) and thrombin receptors (F2r/2 and F2r/3), suggests a possible effect of OPP in clearing blood clots from the circulation via the spleen.

indicates the relationship of one protein to another. A network analysis may thus help to detect other possible proteins affected by the treatment. For example, a number of coagulation factors $(F 2, F 5, F 8, F 9, F 11)$ was found to be connected to the genes significantly upregulated in the spleen blood coagulation network.
These genes might thus be up-regulated and contribute to the blood coagulation process observed in this experiment as well, although the statistical threshold used might not result in these genes being selected as significantly changed. A network analysis can also help in the identification of central regulators in specific networks. 
For example, the $V w f$ gene appeared to be the central regulator in the blood coagulation network, and might thus play a central role in this process by up-regulating the other genes in response to OPP treatment.

In addition to blood coagulation, other functions significantly up-regulated in the spleen by OPP include actin binding, calcium ion binding, cell adhesion, fatty acid metabolism, focal adhesion, prostaglandin synthesis regulation, sugar binding and tumour necrosis factor binding. Genes down-regulated in the spleen on the other hand, were involved in DNA replication, interleukin-9 signalling, nitrogen metabolism and unfolded protein binding.

\section{Gene expression changes in the heart}

In the heart, genes up-regulated by OPP were involved in acetyl-CoA, butanoate, fatty acid and lipid metabolisms, golgi organisation and biogenesis, glutathione metabolism, magnesium ion binding, nitric oxide mediated signal transduction and translation factors. Down-regulated genes in the heart include those involved in energy production, such as the tricarboxylic acid (TCA) cycle (Figure 5A) and the electron transport chain (Figure 5B). Genes involved in protein biosynthesis such as chromatin assembly or disassembly, haem biosynthesis, mitochondrial genome maintenance, mRNA processing and binding, as well as those encoding ribosomal proteins, were also down-regulated.

In the heart TCA cycle, a key enzyme, pyruvate dehydrogenase kinase isoenzyme $4(P d k 4)$, which inhibits pyruvate dehydrogenase and thus minimises carbohydrate oxidation by preventing the flow of glycolytic products into the TCA cycle, was up-regulated. In conjunction with this, the expression of other genes involved in the heart TCA cycle, such as those which produce NADH $(M d h 1)$ and $\mathrm{FADH}_{2}(S h d b)$, were down-regulated as well. Suclg1 and Sucla2, genes encoding subunits of succinate-CoA ligases which convert guanosine triphosphate/adenosine triphosphate (GTP/ ATP) to guanosine diphosphate/adenosine diphosphate (GDP/ADP) were also down-regulated.

In the heart electron transport chain, Ucp3, a gene encoding an uncoupling protein which protects against mitochondrial oxidative damage by reducing the production of ROS, was up-regulated as well. On the other hand, genes in the electron transport chain which encode proteins in Complex I (Ndufb2, Ndufb4, Ndufb10, Ndufc2, Ndufs4), Complex II (Sdhb) as well as those in Complex V (Atp5fi, Atp5j, Atp5k, Atp6v0c, Atp6v1d), were down-regulated.

\section{Real-time qRT-PCR validation}

To confirm the microarray results, the expression levels of six target genes (Table 2) were measured using real- time quantitative reverse transcription-polymerase chain reaction (qRT-PCR). Expression levels of these target genes were normalised to the geometric mean of three housekeeping genes, Sfrs9, Guk1 and Hnrpab. The direction and magnitude of fold changes of the target genes obtained from the real-time qRT-PCR technique were comparable to those obtained from the microarray technique (Figure 6A). Correlation of fold changes obtained by the two gene expression profiling techniques was high $\left(R^{2}=0.9653\right)$ (Figure $\left.6 B\right)$, thus indicating that the microarray data obtained were valid.

\section{Discussion}

As OPP displayed significant antioxidant activities and conferred positive outcomes in various animal models of degenerative diseases without causing toxicity [21-23], we hypothesised that it might also have significant biological effects in animals during healthy states. Thus, we fed BALB/c mice a normal diet with OPP for six weeks and looked for signs of toxicity to confirm that OPP is safe. Microarray gene expression analysis was then carried out on three major organs, the liver, spleen and heart. This study thus represents an important step towards understanding the mode of action of OPP during healthy states, before further studies are carried out to identify the molecular mechanisms involved during diseased states.

\section{OPP up-regulated fatty acid beta oxidation genes and down-regulated cholesterol biosynthesis genes in the liver}

In identifying the in vivo gene expression changes caused by any dietary intervention via microarray analysis, the liver naturally becomes the target organ of interest $[27,28]$. This is because it is a vital organ for various metabolic functions, including detoxification and production of biochemicals necessary for digestion and maintenance of body processes. In this study, functional enrichment analysis on the microarray data from the liver showed that OPP up-regulated several pathways, including lipid catabolism (fatty acid beta oxidation), complement activation, protein biosynthesis and transport, ion and electron transport as well as translation factors.

As the liver is an important site for fatty acid beta oxidation, up-regulation of hepatic lipid catabolism may contribute to the suppression of liver fat and visceral fat accumulation. Stocker and Keaney (2004) suggested that removal of lipids from the body through fatty acid beta oxidation may prevent lipid peroxidation which contributes to atherosclerosis [29]. Interestingly, enhanced hepatic fatty acid synthesis and reduced fatty acid oxidation have also been implied in the development of alcohol-induced fatty liver [30-33]. Thus, OPP may also be 


\section{A}

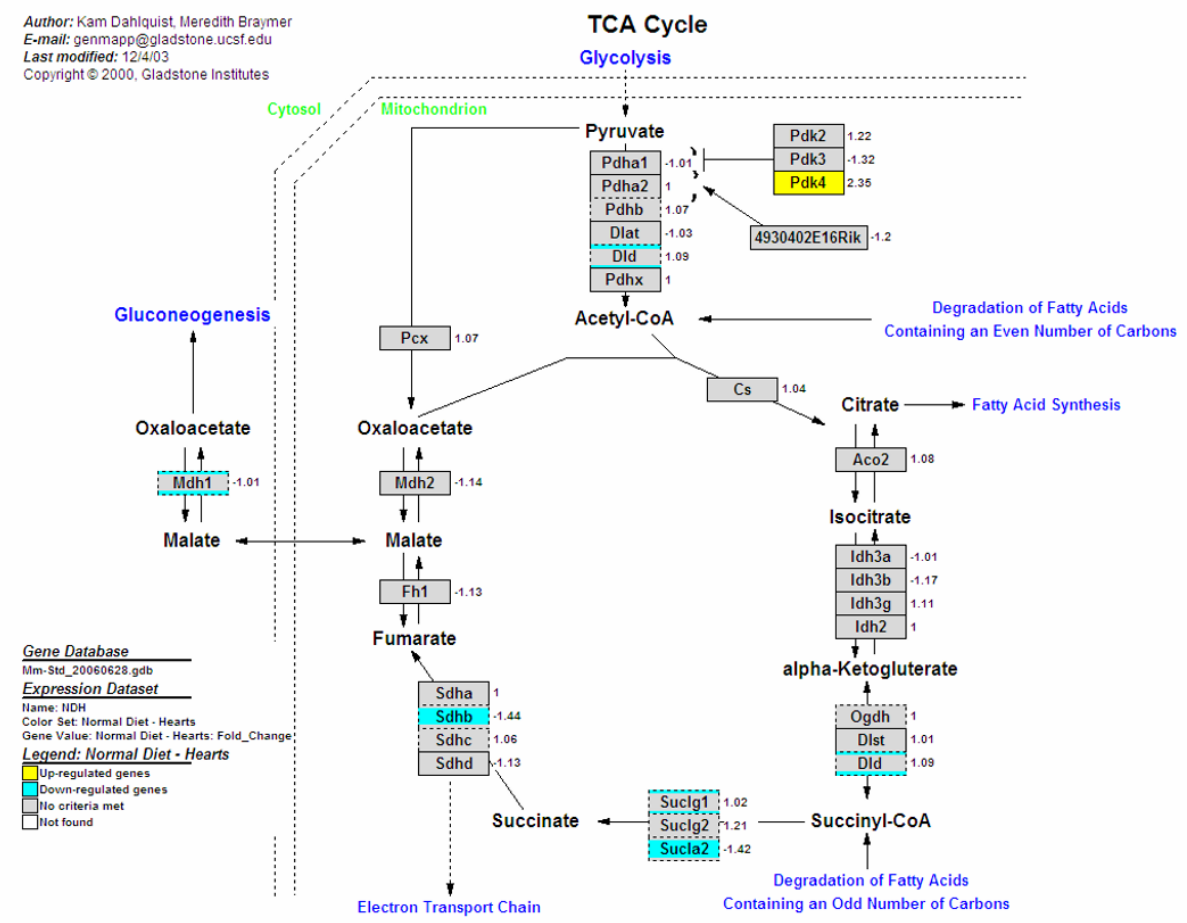

B

Electron Transport Chain

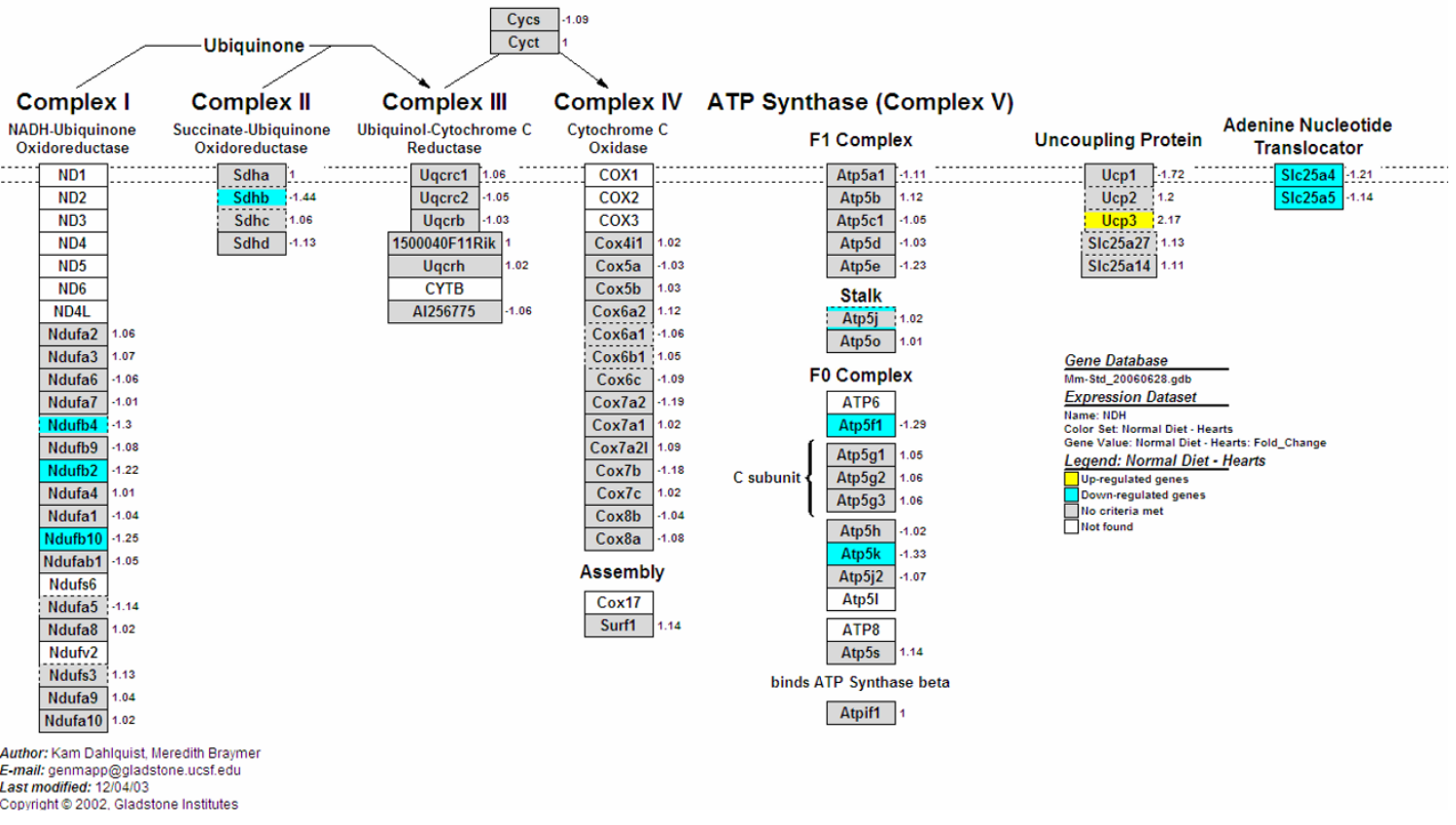

Figure 5 GenMAPPs showing functions and genes significantly changed by OPP in the heart. (A) Genes up-regulated and downregulated in the heart tricarboxylic acid (TCA) cycle. Pdk4, which is involved in fuel selection in the heart by inhibiting pyruvate dehydrogenase and preventing the metabolic shift in ageing hearts from fatty acid beta oxidation towards glycolysis, was up-regulated, while other genes such as Mdh1, Shdb, Suclg1, Sucla2 and Dld were down-regulated. (B) Ucp3, a gene encoding an uncoupling protein which protects against mitochondrial oxidative damage by reducing the production of reactive oxygen species (ROS), was up-regulated, while other genes which encode adenine nucleotide translocators and proteins in Complex I, Complex II and Complex V in the electron transport chain were downregulated. 
Table 2 Genes selected for the real-time qRT-PCR validation experiments

\begin{tabular}{ccccc}
\hline Organ & Symbol & Definition & Accession & Assay ID \\
\hline Liver & Cyp3a11 & Mus musculus cytochrome P450, family 3, subfamily a, polypeptide 11 & NM_007818 & Mm00731567_m1 \\
Liver & Hmgcs1 & Mus musculus 3-hydroxy-3-methylglutaryl-Coenzyme A synthase 1 & NM_145942 & Mm00524111_m1 \\
Spleen & Vwf & Mus musculus Von Willebrand factor homologue & NM_011708 & Mm00550376_m1 \\
Spleen & Brca1 & Mus musculus breast cancer 1 & NM_009764 & Mm00515386_m1 \\
Heart & Pdk4 & Mus musculus pyruvate dehydrogenase kinase, isoenzyme 4 & NM_013743 & Mm00443325_m1 \\
Heart & Rad21 & Mus musculus RAD21 homologue (S. pombe) & NM_009009 & Mm00485474_m1 \\
All & Sfrs9 & Mus musculus splicing factor, arginine/serine rich 9 & NM_025573 & Mm00470546_m1 \\
All & Guk1 & Mus musculus guanylate kinase 1 & NM_008193 & Mm00433888_m1 \\
All & Hnrpab & Mus musculus heterogeneous nuclear ribonucleoprotein A/B & NM_010448 & Mm00468938_m1 \\
\hline
\end{tabular}

These six target genes were selected based on their presence in significant functions, their differential scores, their detection levels, their presence as single splice transcripts in microarrays and their availability as Taqman assays designed across splice junctions. Sfrs9, Guk1 and Hnrpab were used as housekeeping genes as their expression levels were found to be quite stable across treatments in each of the organs tested.

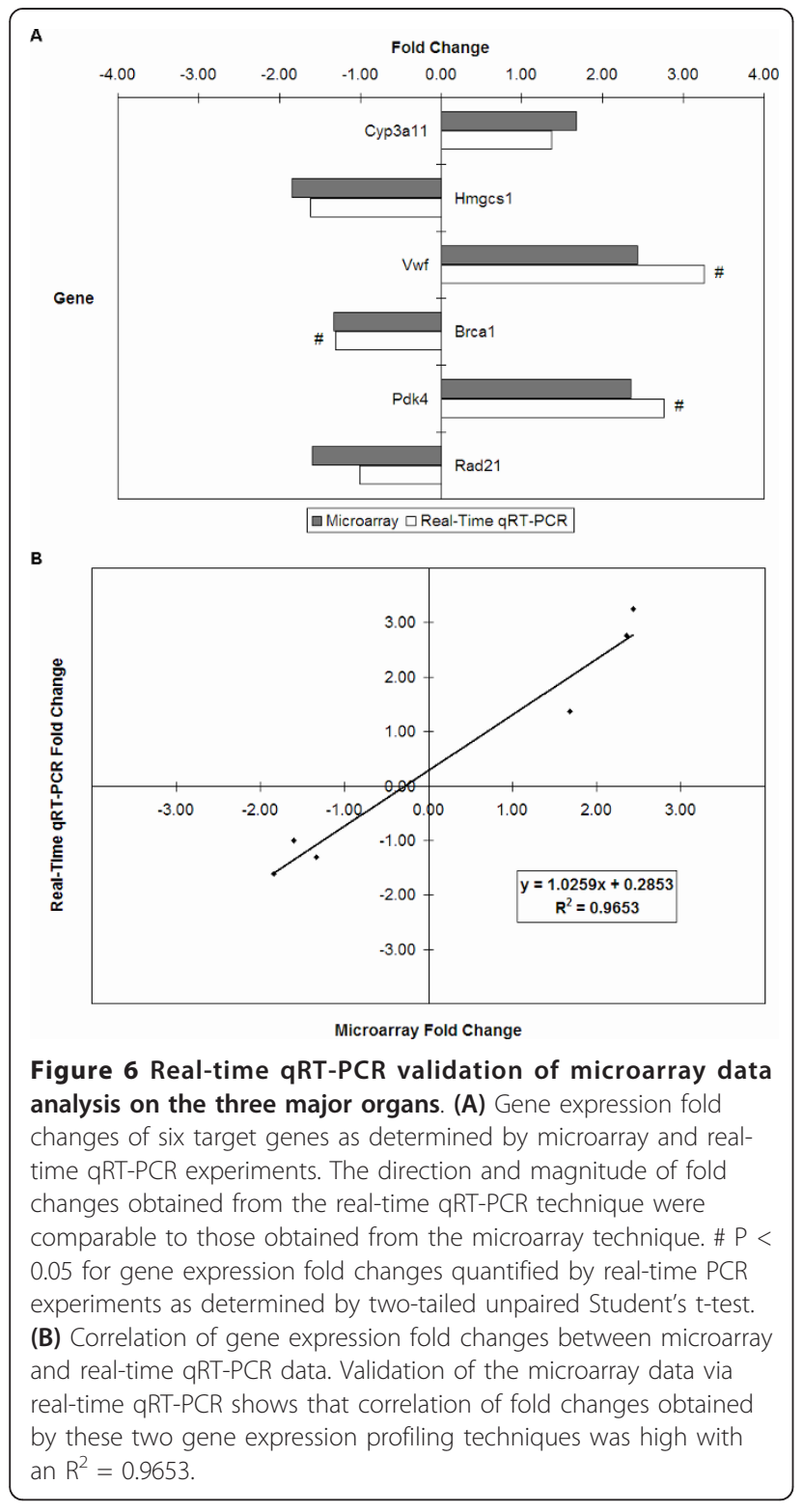

able to reduce atherosclerosis and prevent alcoholinduced liver damage based on its ability to up-regulate hepatic fatty acid beta oxidation. Increased expression of hepatic genes involved in lipid catabolism has also been shown to be effected by the catechins of green tea [34] and the chlorogenic acids of coffee [35].

The up-regulation of genes involved in complement activation observed in the present study, is similar to that reported for resveratrol [36]. In the resveratrol study, complement pathways, both classical and alternative, as well as acute inflammatory response, were upregulated for reasons unknown, although the authors noted that no evidence of widespread inflammatory response was observed. The induction of the classical complement pathway by OPP may be triggered by antibodies, as plant antioxidants were reported to enhance immunity [37]. As an important component of innate immunity, complement activation is necessary for protection against numerous microbial infections $[38,39]$.

Genes involved in ion and electron transport such as those encoding cytochromes P450 and NADH dehydrogenase (ubiquinone) $\mathrm{Fe}-\mathrm{S}$ proteins were also up-regulated by OPP. In general, phenolics may be regarded as xenobiotics by animal cells and are to some extent treated as such through interaction with phase I and phase II detoxification enzyme systems [40]. Phase I detoxification in the liver involves the activation of a series of enzymes called the cytochrome P450 mixed-function oxidases. These enzymes begin the biotransformation process by oxidising, reducing or hydrolysing toxins, thus creating biotransformed intermediates [41]. A phase I detoxification gene of special interest is Cyp3a4, which encodes the most abundant cytochrome P450 in the human liver (around 30\%), responsible for $60 \%$ of cytochrome P450-mediated metabolism of drugs in therapeutic use [42]. Cyp3a4 was found to be induced more than 2-fold by dietary flavonoids such as quercetin and grape seed extract in HepG2 hepatocytes [42]. Cyp3a11 is the mouse homologue of the human Cyp3a4 [43]. It 
is interesting to note that OPP up-regulated Cyp $3 a 11$ 1.68 -fold in the mouse liver. Incidentally, Cyp3a11 was up-regulated in the mouse liver as well by $\alpha$-tocopherol [44]. This indicates that OPP has similar effects to other dietary antioxidants and may thus be involved in drugherbal/botanical interaction effects. Phase II detoxification enzymes such as UDP glycosyltransferases perform conjugation reactions which help to convert biotransformed intermediates into less toxic, water-soluble substances that are easily excreted or eliminated from the body [41]. In the present study, genes Ugt1a6 and Ugt1a9 encoding UDP glycosyltransferases were upregulated 2.11-fold and 1.95-fold respectively. Comt, the gene which encodes catechol-O-methyltransferase, an enzyme involved in tyrosine metabolism, was also upregulated by OPP. Catechol-O-methyltransferase has been found to be involved in the metabolism of hydroxytyrosol, the major component of olive phenolics [45]. The up-regulated translation factors involved in protein synthesis, may also reflect the production of extra enzymes which help in metabolising OPP.

Other genes up-regulated by OPP are those encoding chemokine (C-X-C motif) ligand 12 ( $\mathrm{Cxcl12}$ ), gap junction membrane channel protein beta $1(G j b 1)$ and androgen receptor $(A r)$, which were 1.87-, 1.38- and 2.30 -fold increased respectively. Cxcl12 is a gene encoding a chemokine modulating the progression of liver fibrosis through its action on hepatic stellate cells [46], while Gjb1 encodes a gap junction membrane channel protein which plays an important role in the regulation of signal transfer and growth control in the liver. The expression of these genes decreased as liver diseases progress to cirrhosis and hepatocellular carcinoma [47]. Both of these genes were down-regulated by comfrey (Cxcl12 down-regulated 5.26-fold, Gjb1 down-regulated 2.08-fold), a herbal plant containing pyrrolizidine alkaloids believed to cause hepatotoxicity in humans and carcinogenicity in animals [46]. Ar was also down-regulated by comfrey (13.6-fold), as well as by riddelliine (19.6-fold) which also contains pyrrolizidine alkaloids [48]. Hence, the up-regulation of these three genes indicates that OPP may have hepatoprotective effects, and also implies that they do not cause hepatotoxicity.

Genes involved in cholesterol biosynthesis on the other hand, were down-regulated by OPP in this study. Cholesterol is an important constituent of cellular membranes and serves as a precursor in the formation of bile acids and steroid hormones. Excessive cholesterol however, is implicated in atherosclerotic lesions and gallstone formation [49]. The reduced expression of genes involved in cholesterol biosynthesis in the mouse liver suggests a possible role of OPP in reducing atherosclerosis and hence, cardiovascular disease. Overall, the gene expression changes caused by OPP in the liver suggest hepatoprotective and anti-dyslipidaemic effects of the extract.

\section{OPP up-regulated blood coagulation genes in the spleen}

In the spleen, the most interesting genes up-regulated by OPP were those involved in blood coagulation. The regulation of these genes suggests that the spleen might be playing an active protective role in improving blood circulation and clearing blood clots from the body. OPP may thus function as anti-thrombotic agents, considering the fact that as a peripheral lymphoid organ, the spleen is also involved in mechanical filtration of the blood to remove unwanted materials [50]. However, this is still hypothetical and further experiments are required to show that OPP helps in clearing and not causing blood clots.

\section{Gene regulation by OPP in the heart showed similarities} with that of caloric restriction

Ageing in the heart involves a transcriptional shift from fatty acid metabolism to carbohydrate metabolism. This shift was also found to differentiate maladaptive hypertrophy (increased glycolysis) from adaptive or exerciseinduced hypertrophy (increased fatty acid beta oxidation) [51]. A key enzyme, pyruvate dehydrogenase kinase isoenzyme $4(P d k 4)$, inhibits pyruvate dehydrogenase and thus minimises carbohydrate oxidation by preventing the flow of glycolytic products into the TCA cycle. Hence, the up-regulation of $P d k 4$ in the heart by OPP may prevent a metabolic shift towards glycolysis and thus ageing. Another gene, Ucp3, encoding an uncoupling protein which protects against mitochondrial oxidative damage by reducing the production of ROS, was up-regulated in the heart by OPP as well. Ucp 3 may transport fatty acids out of the mitochondria, thereby protecting the mitochondria from fatty acid anions or peroxides [52]. Besides up-regulating $P d k 4$ and $U_{c p} 3$, OPP also up-regulated another seven genes in the heart, carnitine acetyltransferase (Crat), catechol-O-methyltransferase (Comt), CCAAT/enhancer binding protein (Cebpb), enoyl-CoA, hydratase/3-hydroxyacyl CoA dehydrogenase (Ehhadh), plasma membrane associated protein (S3-12), solute carrier family 27 (fatty acid transporter) (Slc27a1) and xanthine dehydrogenase $(X d h)$. Lee et al. (2002) demonstrated the down-regulation of these nine genes in the hearts of ageing $\mathrm{B} \mathrm{CC}_{3} \mathrm{~F}_{1}$ mice [53]. Six of these genes, Pdk4, Ucp3, Comt, Cebpb, S3-12 and $X d h$ were significantly up-regulated by caloric restriction, thus opposing the effects of ageing [53]. Additional genes up-regulated by OPP and also by caloric restriction in the heart include carbonic anhydrase 4 (Car4), carboxylesterase 3 (Ces3), purine-nucleoside phosphorylase (Pnp) and tumour protein D52-like 1 
(Tpd52l1) [53]. Hence, a role for OPP in preventing or slowing down ageing of the heart is implicated.

It was also noted that genes up-regulated in the hearts of ageing $\mathrm{B} \mathrm{CC}_{3} \mathrm{~F}_{1}$ mice such as aminolevulinic acid synthase 1 (Alas1), mature T cell proliferation 1 (Mtcp1) and protein tyrosine phosphatase 4a2 (Ptp4a2) [53], were downregulated by OPP in the heart in the present study. In the group that had caloric restriction on the other hand, cyclin D2 (Ccnd2), secreted acidic cysteine rich glycoprotein (Sparc) and transferrin receptor (Tfrc) genes were downregulated [53]. In the present study, these three genes were also down-regulated by OPP. It has been found that caloric restriction confers rapid positive effects on the heart, by rapidly shifting gene expression towards a state of reduced cardiac remodelling and fibrosis as well as enhanced contractility [54]. Several plant phenolics such as resveratrol, quercetin, butein and piceatannol have been proposed as potential caloric restriction mimetics [1]. The regulation of genes by OPP in the heart suggests that they may also act as caloric restriction mimetics.

In addition to genes similarly affected by caloric restriction, antioxidant genes such as those encoding erythroid derived-nuclear factor 2-like 1 ( $N f e 2 l 1$ ), glutamate-cysteine ligase (Gclm) and various glutathione Stransferases (Gstm2, Gstm5, Gstm 6) were up-regulated. Glutathione S-transferases are important antioxidant enzymes essential in the detoxification of carcinogens and scavenging of ROS $[40,55,56]$. The up-regulation of these antioxidant genes thus indicates that OPP provides a higher antioxidant defence in the heart, which is an organ prone to damage by prooxidants.

The expression levels of genes in the heart TCA cycle, such as those encoding proteins which produce NADH $(M d h 1)$ and $\mathrm{FADH}_{2}(S h d b)$, were also down-regulated. Suclg1 and Sucla2, genes encoding subunits of succinate-CoA ligases which convert GTP/ATP to GDP/ADP were also down-regulated. Finally, genes in the electron transport chain which encode proteins in Complex I, Complex II and Complex V were down-regulated as well. Electrons derived from metabolic reducing equivalents (NADH and $\mathrm{FADH}_{2}$ ) are fed into the electron transport chain through either Complex I or Complex II, and eventually pass to molecular oxygen to form water in Complex IV, while ATP is being formed in Complex V. These results imply that energy production in the heart was down-regulated by OPP. Hence, in reducing the amount of energy generated, OPP may act as antioxidants in reducing the amount of ROS produced in the heart, suggesting a beneficial effect of the extract in preventing cardiac oxidative stress.

\section{Summary and implications of the study}

The discovery of OPP opens up a niche for value-added novel nutraceuticals. In this study, we confirmed that
OPP was not toxic to mice as these compounds did not significantly alter the organ histology of the animals as well as their haematology and clinical biochemistry parameters. In addition, this study identified the global gene expression profiles caused by OPP in three major organs of mice on a normal diet. Supplementation of OPP to these mice resulted in numerous beneficial biological activities during healthy states. In livers of these mice, lipid catabolism genes were up-regulated while cholesterol biosynthesis genes were down-regulated, suggesting that OPP may find potential applications as hepatoprotective and anti-dyslipidaemic supplements. OPP may also act as possible anti-thrombotic agents since they encouraged the aggregation of platelets in spleens. OPP also retarded the ageing process in hearts, similar to the effects of caloric restriction.

Although the clinical biochemistry parameters on lipid profiles (triglycerides, total cholesterol, low-density lipoproteins and high-density lipoproteins) measured in this study did not show any significant differences between the groups, mice in the treatment group did show a delay in weight gain throughout the six weeks of feeding compared to the control group. Also, while the blood serum lipid profiles measured in the present study did not show any significant differences between the control and treated mice even though the hepatic gene expression profiles had changed, a similar observation was made by Matsui et al. (2005) when cocoa was given to mice [57]. In this previous study, cocoa ingestion increased total cholesterol concentrations in the blood sera, whereas body weight gain and hepatic cholesterol biosynthesis were decreased [57]. Hence, the difference between the clinical biochemistry parameters and the hepatic gene expression changes observed in the present study might be caused by other factors, which include reabsorption of cholesterol in the small intestine or other roles that the small intestine may play in the aetiology of obesity [58]. Perhaps the lipid profiles of livers in addition to blood sera should be conducted in future studies in order to directly correlate these profiles with the hepatic gene expression changes observed.

To our knowledge, this is the first study in which microarray analysis has been carried out on major organs of mice supplemented with OPP. In identifying possible pharmacological, nutraceutical or toxicological responses, the biological representation of a set of genes is more interesting than the genes themselves. By using different microarray softwares for statistical and functional analyses, we identified genes, gene ontologies, pathways and networks that were significantly changed by OPP in different major organs of mice on a normal diet. The gene expression profiles obtained indicate several mechanisms as to how OPP exerts its beneficial effects in vivo. These findings implicate broader 
pleiotropic effects of OPP than previously expected. This study also serves as a model for the dissection of many other complex responses mediated by dietary phenolics and plant phytochemicals in general.

\section{Conclusions}

Our study was designed to understand the global functional profiles of OPP in mice which were on a normal diet. This analysis shows some of the molecular mechanisms as to how OPP exerts biological activities in vivo during healthy states. It thus provides a background for further hypotheses to be tested in future experiments involving OPP specifically, and dietary phenolics in general. This study also sets the scene for identifying the molecular modes of action involved in conferring the positive biological effects of OPP during various diseased states.

\section{Methods \\ OPP samples}

The OPP samples used in this study were prepared according to the methods described in Sambanthamurthi et al. (2008) [19]. OPP contains numerous phenolic acids, with three isomers of caffeoylshikimic acid as major components [20,21]. Other phenolic acids present include protocatechuic acid and $p$-hydroxybenzoic acid. The detailed composition of OPP is as described earlier [21].

\section{Animals, diets and treatments}

All male inbred BALB/c mice which were designated for this study $(n=20)$ were purchased from the Institute of Medical Research, Kuala Lumpur, Malaysia, at around five weeks of age just after weaning. All animal procedures were approved by the Animal Care and Use Committee of the University of Malaya, Kuala Lumpur, Malaysia. The animals were randomly assigned into cages ( $n=5$ per cage) and acclimatised for one week, during which a standard chow diet purchased from the University of Malaya and distilled water, were given. At the start of the experiment, the diet of the animals was changed to a custom-made normal diet $(58.2 \% \mathrm{kcal} / \mathrm{kcal}$ carbohydrate, $27.2 \% \mathrm{kcal} / \mathrm{kcal}$ protein and $14.6 \% \mathrm{kcal} /$ kcal fat, including cellulose, mineral mix, vitamin mix and DL-methionine).

The control group $(n=10)$ was supplemented with distilled water while the treatment group $(n=10)$ was supplemented with OPP, as drinking fluids ad libitum. The antioxidant content of the OPP given was 1500 ppm gallic acid equivalent. Food and fluids were changed daily. During the animal feeding process, body weights were monitored every week, while fluid intake was monitored every day, for six weeks. Food intake and faecal output were monitored for seven consecutive days between week two to week three.

After six weeks, the mice were sacrificed via euthanasia with diethyl ether followed by exsanguination. Blood samples were collected after an overnight food fast (with fluids still provided) via cardiac puncture, while three major organs including livers, spleens and hearts were excised, rinsed in $0.9 \% \mathrm{w} / \mathrm{v}$ sodium chloride solution and weighed. Half of the organs $(n=5)$ were preserved in $10 \%$ formalin diluted with phosphate buffered saline for histology analysis, while the other half $(n=5)$ were snap-frozen in liquid nitrogen and stored at $-80^{\circ} \mathrm{C}$ until the total RNA extraction process for gene expression analysis.

\section{Haematology analysis on blood samples}

About $200 \mu \mathrm{L}$ from half of each blood sample collected $(n=4)$ was aliquoted into a blood collection tube containing ethylenediaminetetraacetic acid to prevent clotting. These whole blood samples were sent immediately after dissection of the animals to the Clinical Biochemistry and Haematology Laboratory, Department of Veterinary Pathology and Microbiology, Faculty of Veterinary Medicine, University of Putra Malaysia, Serdang, Selangor, Malaysia, for haematology analysis. The analysis was carried out using the Animal Blood Counter Vet Haematology Analyser (Horiba ABX, France).

\section{Clinical biochemistry analysis on blood samples}

In order to obtain sera, blood samples were allowed to clot at room temperature for two hours before being centrifuged at $1000 x g$ for five minutes, after which the supernatant layers were collected and stored at $-20^{\circ} \mathrm{C}$. The blood serum samples obtained were then sent for clinical biochemistry analysis using the Roche/Hitachi 902 Chemistry Analyser (Roche/Hitachi, Switzerland) in the Clinical Biochemistry and Haematology Laboratory, Department of Veterinary Pathology and Microbiology, Faculty of Veterinary Medicine, University of Putra Malaysia. Clinical biochemistry parameters which were examined include alanine aminotransferase, aspartate aminotransferase, glucose, serum total protein, albumin, globulin, ratio of albumin to globulin, total cholesterol, triglycerides, low-density lipoproteins and high-density lipoproteins. Of a total of ten serum samples per group obtained for clinical biochemistry analysis, two samples in the control group and three samples in the treatment group were excluded due to blood lysis.

\section{Histology analysis on organs}

Histology analysis on dissected organs $(n=5)$ preserved in $10 \%$ buffered formalin was carried out in the Department of Pathology, Faculty of Medicine, University of 
Malaya, using standard routine procedures with haematoxylin and eosin staining.

\section{Total RNA extraction for gene expression analysis}

All precautions in handling RNA were taken in this study. Total RNA isolation from mouse organs $(n=5)$ was carried out using the RNeasy Mini Kit (Qiagen, Inc., Valencia, CA) and QIAshredder homogenizers (Qiagen, Inc., Valencia, CA), preceded by grinding in liquid nitrogen using mortars and pestles. The total RNA samples obtained were subjected to NanoDrop 1000A Spectrophotometer (Thermo Fisher Scientific, Waltham, MA) measurement for yield and purity assessment. Integrity of the total RNA samples was assessed using the Agilent 2100 Bioanalyzer (Agilent Technologies, Santa Clara, CA) and Agilent RNA 6000 Nano Chip Assay Kit (Agilent Technologies, Santa Clara, CA). Four total RNA samples with the highest RNA Integrity Numbers and 28S/18S rRNA ratios within each condition (either control or treatment) were then selected for microarray studies.

\section{Microarray hybridisation, washing and scanning}

Amplification of total RNA samples which were of high yield, purity and integrity was carried out using the Illumina TotalPrep RNA Amplification Kit (Ambion, Inc., Austin, TX). The cRNA produced was then hybridised to the Illumina MouseRef-8 Version 1 Expression BeadChip (Illumina, Inc., San Diego, CA), using the Direct Hybridization Kit (Illumina, Inc., San Diego, CA). Microarray hybridisation, washing and scanning were carried out according to the manufacturer's instructions. The raw gene expression data obtained are available at Gene Expression Omnibus [59] (Accession number: GSE28824).

\section{Microarray data analysis}

Quality control of the hybridisation, microarray data extraction and initial analysis were carried out using the Illumina BeadStudio software (Illumina, Inc., San Diego, CA). Outlier samples were removed via hierarchical clustering analysis provided by the Illumina BeadStudio software and also using the TIGR MeV software (Institute for Genomic Research, Rockville, MD) [60], via different distance metrics. A minimum of three biological replicates per condition (with outliers removed) was then considered for further analysis. For livers, four replicates in the control group and three replicates in the treatment group were analysed. For spleens and hearts respectively, four replicates in the control group and four replicates in the treatment group were analysed.

Gene expression values were normalised using the rank invariant method and genes which had Detection
Levels of more than 0.99 in either the control or treatment samples were considered significantly detected. To filter the data for genes which changed significantly in terms of statistics, the Illumina Custom error model was used and genes were considered significantly changed at a |Differential Score| of more than 20, which was equivalent to a P Value of less than 0.01 [61].

The genes and their corresponding data were then exported into the Microsoft Excel software (Microsoft Corporation, Richmond, WA) for further analysis. To calculate fold changes, an arbitrary value of 10 was given to expression values which were less than 10 . Fold changes were then calculated by dividing the mean values of Signal Y (treatment) with those of Signal $x$ (control) if the genes were up-regulated and vice versa if the genes were down-regulated. Two-way (gene and sample) hierarchical clustering of the significant genes was then performed using the TIGR MeV software to ensure that the replicates of each condition were clustered to each other. The Euclidean distance metric and average linkage method were used to carry out the hierarchical clustering analysis.

Changes in biological pathways and gene ontologies were assessed via functional enrichment analysis, using the GenMAPP [24] and MAPPFinder [25] softwares (University of California at San Francisco, San Francisco, CA). The MAPPFinder software ranks GenMAPPs (pathways) and gene ontologies based on the hypergeometric distribution. Readers are referred to Doniger et al. (2003) [25] for further explanations of the terms used in the MAPPFinder software. GenMAPPs and gene ontologies which had Permuted P Values of less than 0.01 , Numbers of Genes Changed of more than or equal to 2 and $\mathrm{Z}$ Scores of more than 2 were considered significant.

It should be noted that the MAPPFinder software clusters multiple probes for a distinct gene into a single gene grouping in order to calculate the number of distinct genes which meet the user-defined criteria, not the probes. In this study, up-regulated and down-regulated genes were analysed separately in the functional enrichment analysis but were viewed together in each GenMAPP. Boxes coloured yellow indicate genes which were up-regulated while those coloured blue indicate genes which were down-regulated. The fold changes are indicated next to the boxes. Individual boxes which have different shadings within them indicate the presence of multiple probes (splice transcripts) within a single gene.

Changes in regulatory networks were also analysed through the use of the Ingenuity Pathways Analysis software (Ingenuity ${ }^{\circledR}$ Systems, Redwood City, CA) [26]. For each organ, a dataset containing differentially expressed genes and their corresponding fold changes was uploaded into the application. Analyses of up-regulated 
and down-regulated genes were carried out separately. Each gene identifier was mapped to its corresponding gene object in the Ingenuity Pathways Knowledge Base. These genes were overlaid onto a global molecular network developed from information contained in the Ingenuity Pathways Knowledge Base. Networks of these focus genes were then algorithmically generated based on their connectivity.

A network is a graphical representation of the molecular relationships between genes or gene products. Genes or gene products were represented as nodes, and the biological relationship between two nodes was represented as an edge (line). The intensity of the node colour indicates the degree of up-regulation (red) or downregulation (green). Nodes were displayed using various shapes that represented the functional class of the gene product. Edges were displayed with various labels that described the nature of the relationship between the nodes.

\section{Real-time qRT-PCR validation}

Two-step real-time qRT-PCR studies were carried out on six target genes selected to represent the different organs used in the microarray experiments, using TaqMan Gene Expression Assays (Applied Biosystems, Foster City, CA). These genes were selected based on their presence in significant functions, their differential scores, their detection levels, their presence as single splice transcripts in microarrays and their availability as Taqman assays designed across splice junctions. The same aliquots of total RNA samples used in the microarray experiments were utilised for this analysis. Primer and probe sets for the selected genes were obtained from the Applied Biosystems Inventoried Assays-OnDemand (Applied Biosystems, Foster City, CA).

Briefly, reverse transcription to generate first-strand cDNA from total RNA was carried out using the HighCapacity cDNA Reverse Transcription Kit (Applied Biosystems, Foster City, CA). Real-time PCR was then carried out on the first-strand cDNA generated using a 25 $\mu \mathrm{L}$ reaction volume in an Applied Biosystems 7000 Real-Time PCR System (Applied Biosystems, Foster City, CA), with the following conditions: $50^{\circ} \mathrm{C}, 2 \mathrm{~min}$ utes, 1 cycle; $95^{\circ} \mathrm{C}, 10$ minutes, 1 cycle; $95^{\circ} \mathrm{C}, 15$ seconds and $60^{\circ} \mathrm{C}, 1$ minute, 40 cycles. For gene expression experiments, reactions for each biological replicate and non-template control (NTC) were carried out in duplicates. For amplification efficiency determination, reactions were carried out in triplicates.

Quality control of the replicates used, real-time qRTPCR data extraction and initial analysis were carried out using the 7000 Sequence Detection System software (Applied Biosystems, Foster City, CA). A manual threshold of 0.6000 and an auto baseline were applied in order to obtain the threshold cycle $(\mathrm{Ct})$ for each measurement taken. The threshold was chosen as it intersected the exponential phase of the amplification plots [62]. The criteria for quality control of the data obtained include $\Delta \mathrm{Ct}$ of less than 0.5 between technical replicates and $\Delta \mathrm{Ct}$ of more than 5.0 between samples and NTCs [63].

Relative quantification of the target genes of interest was carried out using the qBase 1.3.5 software (Center for Medical Genetics, Ghent University Hospital, Ghent, Belgium) [64], which takes into account the calculations of amplification efficiencies and multiple housekeeping genes. Expression levels of target genes were normalised to the geometric mean of three housekeeping genes, arginine/serine rich splicing factor 9 (Sfrs 9 ), guanylate kinase $1(G u k 1)$ and heterogeneous nuclear ribonucleoprotein A/B (Hnrpab). These genes were chosen as they were shown to be stable across the previously obtained microarray data, and confirmed to be more stable than $18 \mathrm{~S}$ ribosomal RNA (18SrRNA), via the usage of the geNorm 3.5 software (Center for Medical Genetics, Ghent University Hospital, Ghent, Belgium) [65].

\section{Statistical analysis}

Statistical analysis was carried out by using the twotailed unpaired Student's t-test available in the Microsoft Excel software (Microsoft Corporation, Redmond, WA) unless otherwise stated. Differences with P Values of less than 0.05 were considered statistically significant.

\section{Additional material}

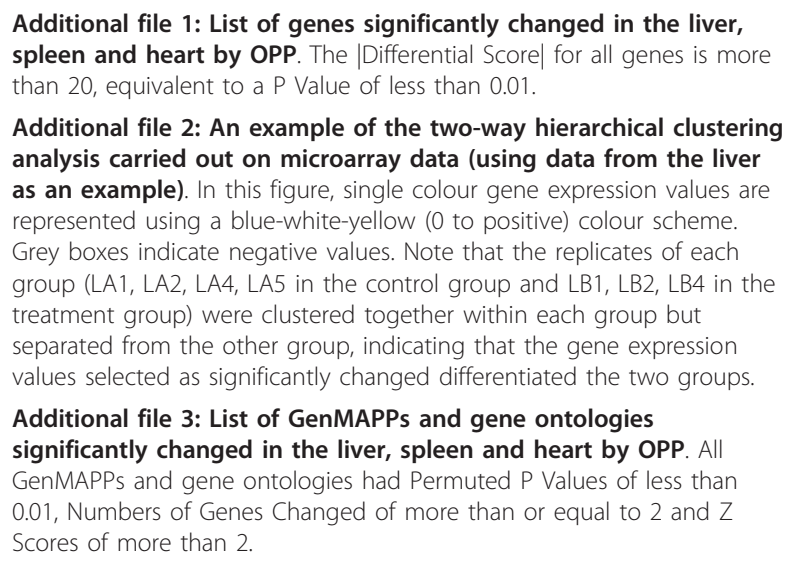

\section{List of abbreviations}

ADP: adenosine diphosphate; ATP: adenosine triphosphate; CCAAT: cytidinecytidine-adenosine-adenosine-thymidine; CoA: coenzyme A; CDNA: complementary deoxyribonucleic acid; CRNA: complementary ribonucleic acid; Ct: threshold cycle; DNA: deoxyribonucleic acid; $\mathrm{FADH}_{2}$ : flavin adenine dinucleotide (fully reduced or hydroquinone form); Fe: iron; GDP: guanosine diphosphate; GenMAPP: Gene Map Annotator and Pathway Profiler; GTP: guanosine triphosphate; HMG: 3-hydroxy-3-methyl-glutaryl; ID: identifier; MAPP: Map Annotator and Pathway Profiler; MAPPFinder: Map Annotator 
and Pathway Profiler Finder; MeV: MultiExperiment Viewer; mRNA: messenger ribonucleic acid; NAD(P): nicotinamide adenine dinucleotide phosphate; NADH: nicotinamide adenine dinucleotide (reduced form); NDH: Normal Diet - Hearts; NDL: Normal Diet - Livers; NTC: non-template control; O: oxygen; OPP: oil palm phenolics; p: para; P: phosphate; PP: pyrophosphate; QRT-PCR: quantitative reverse transcription-polymerase chain reaction; RNA: ribonucleic acid; ROS: reactive oxygen species; rRNA: ribosomal ribonucleic acid; S: sulphur; s.e.m.: standard error of the mean; TCA: tricarboxylic acid; TIGR MeV: The Institute for Genomic Research; UDP: uridine diphosphate

\section{Acknowledgements}

The authors thank Hajah Che Anishas Che Idris (Malaysian Palm Oil Board) for assistance in animal care and handling, and Dr Ong Kien Chai (University of Malaya) for assistance in histology analysis. This study was an integral part of the PhD program of SSL, who was supported by the Graduate Student Assistantship Scheme of the Malaysian Palm Oil Board. This research was fully funded by and carried out at the Malaysian Palm Oil Board.

\section{Author details}

'Malaysian Palm Oil Board, No. 6, Persiaran Institusi, Bandar Baru Bangi, 43000 Kajang, Selangor, Malaysia. ${ }^{2}$ University of Malaya, 50603 Kuala Lumpur, Malaysia. ${ }^{3}$ Malaysian Palm Oil Council, 2nd Floor, Wisma Sawit, Lot 6, SS6, Jalan Perbandaran, 47301 Kelana Jaya, Selangor, Malaysia.

\section{Authors' contributions}

SSL carried out the animal feeding, collected the samples, carried out the histology, microarray and real-time qRT-PCR experiments, performed the data analysis, interpreted the data and drafted the manuscript. SDS helped in the interpretation of the microarray data, in addition to supervising the study. KS designed the animal feeding. YAT was involved in the preparation of OPP. RS conceived the study and participated in its design and coordination, in addition to supervising the study. All authors participated in helpful discussions and read as well as approved the final manuscript.

Received: 8 June 2011 Accepted: 25 August 2011

Published: 25 August 2011

\section{References}

1. Fontana L, Klein S: Aging, adiposity, and calorie restriction. Jama 2007, 297:986-994

2. World Health Organization: Chronic Diseases. [http://www.who.int/topics/ chronic_diseases/en/].

3. Young IS, Woodside JV: Antioxidants in health and disease. J Clin Pathol 2001, 54:176-186.

4. Sen CK, Packer L: Antioxidant and redox regulation of gene transcription. Faseb J 1996, 10:709-720

5. Kaeberlein M, McDonagh T, Heltweg B, et al: Substrate-specific activation of sirtuins by resveratrol. J Biol Chem 2005, 280:17038-17045

6. Tsao R, Deng Z: Separation procedures for naturally occurring antioxidant phytochemicals. J Chromatogr B Analyt Technol Biomed Life Sci 2004, 812:85-99.

7. Wattanapenpaiboon N, Wahlqvist MW: Phytonutrient deficiency: The place of palm fruit. Asia Pac J Clin Nutr 2003, 12:363-368.

8. Narayana KR, Reddy MS, Chaluvadi MR, et al: Bioflavonoids classification, pharmacological, biochemical effects and therapeutic potential. Indian Journal of Pharmacology 2001, 33:2-16.

9. Kaliora AC, Dedoussis GV, Schmidt H: Dietary antioxidants in preventing atherogenesis. Atherosclerosis 2006, 187:1-17.

10. Gohil K, Chakraborty AA: Applications of microarray and bioinformatics tools to dissect molecular responses of the central nervous system to antioxidant micronutrients. Nutrition 2004, 20:50-55.

11. Iida K, Nishimura I: Gene expression profiling by DNA microarray technology. Crit Rev Oral Biol Med 2002, 13:35-50.

12. de Boer VC, van Schothorst EM, Dihal AA, et al: Chronic quercetin exposure affects fatty acid catabolism in rat lung. Cell Mol Life Sci 2006, 63:2847-2858.

13. Raskin I, Ripoll C: Can an apple a day keep the doctor away? Curr Pharm Des 2004, 10:3419-3429.
14. Van Erk MJ, Teuling E, Staal YC, et al: Time- and dose-dependent effects of curcumin on gene expression in human colon cancer cells. J Carcinog 2004, 3:8.

15. Yang SP, Wilson K, Kawa A, et al: Effects of green tea extracts on gene expression in HepG2 and Cal-27 cells. Food Chem Toxicol 2006, 44:1075-1081.

16. Sambanthamurthi R, Sundram K, Tan Y: Chemistry and biochemistry of palm oil. Prog Lipid Res 2000, 39:507-558.

17. Sundram K, Sambanthamurthi $R$, Tan YA: Palm fruit chemistry and nutrition. Asia Pac J Clin Nutr 2003, 12:355-362.

18. Tan YA, Sambanthamurthi R, Sundram K, et al: Valorisation of palm byproducts as functional components. Eur J Lipid Sci Technol 2007, 109:380-393.

19. Sambanthamurthi $R$, Tan YA, Sundram K: Treatment of vegetation liquors derived from oil-bearing fruit. United States Patent US 7387802 B2: Malaysian Palm Oil Board; 2008.

20. Sambandan TG, Rha CK, Sinskey AJ, et al: Composition comprising caffeoylshikimic acids, protocatechuic acid, hydroxytyrosol, hydroxybenzoic acid and their derivatives and method of preparation thereof. World Patent Application Publication WO 2010137943: Malaysian Palm Oil Board; 2010.

21. Sambanthamurthi $R$, Tan $Y A$, Sundram $K$, et al: Oil palm vegetation liquor: A new source of phenolic bioactives. Br J Nutr 2011.

22. Sambanthamurthi R, Tan YA, Sundram $K$, et al: Positive outcomes of oil palm phenolics on degenerative diseases in animal models. Br J Nutr 2011.

23. Sekaran SD, Leow SS, Abobaker $N$, et al: Effects of oil palm phenolics on tumor cells in vitro and in vivo. African Journal of Food Science 2010, 4:495-502.

24. Dahlquist KD, Salomonis N, Vranizan K, et al: GenMAPP, a new tool for viewing and analyzing microarray data on biological pathways. Nat Genet 2002, 31:19-20

25. Doniger SW, Salomonis N, Dahlquist KD, et al: MAPPFinder: Using Gene Ontology and GenMAPP to create a global gene-expression profile from microarray data. Genome Biol 2003, 4:R7.

26. Ingenuity Pathways Analysis. [http://www.ingenuity.com/].

27. Berger A, Roberts MA, Hoff B: How dietary arachidonic- and docosahexaenoic- acid rich oils differentially affect the murine hepatic transcriptome. Lipids Health Dis 2006, 5:10.

28. Xu Z, Le K, Moghadasian MH: Long-term phytosterol treatment alters gene expression in the liver of apo E-deficient mice. J Nutr Biochem 2008, 19:545-554.

29. Stocker R, Keaney JF Jr: Role of oxidative modifications in atherosclerosis. Physiol Rev 2004, 84:1381-1478.

30. Feinman L, Lieber CS: Ethanol and lipid metabolism. Am J Clin Nutr 1999 70:791-792.

31. You M, Fischer $M$, Deeg $M A$, et al: Ethanol induces fatty acid synthesis pathways by activation of sterol regulatory element-binding protein (SREBP). J Biol Chem 2002, 277:29342-29347.

32. You M, Crabb DW: Recent advances in alcoholic liver disease II. Minireview: Molecular mechanisms of alcoholic fatty liver. Am J Physiol Gastrointest Liver Physiol 2004, 287:G1-6.

33. Yin $H Q$, Kim M, Kim JH, et al: Differential gene expression and lipid metabolism in fatty liver induced by acute ethanol treatment in mice. Toxicol Appl Pharmacol 2007, 223:225-233.

34. Murase T, Nagasawa A, Suzuki J, et al: Beneficial effects of tea catechins on diet-induced obesity: Stimulation of lipid catabolism in the liver. Int $J$ Obes Relat Metab Disord 2002, 26:1459-1464.

35. Shimoda H, Seki E, Aitani M: Inhibitory effect of green coffee bean extract on fat accumulation and body weight gain in mice. BMC Complement Altern Med 2006, 6:9.

36. Baur JA, Pearson KJ, Price NL, et al: Resveratrol improves health and survival of mice on a high-calorie diet. Nature 2006, 444:337-342.

37. Lampe JW: Health effects of vegetables and fruit: Assessing mechanisms of action in human experimental studies. Am J Clin Nutr 1999, 70:475S-490S.

38. Abbas AK, Lichtman AH: Cellular and Molecular Immunology. 5 edition. Philadelphia: Elsevier Saunders; 2005.

39. Jayasekera JP, Moseman EA, Carroll MC: Natural antibody and complement mediate neutralization of influenza virus in the absence of prior immunity. J Virol 2007, 81:3487-3494. 
40. Moskaug JO, Carlsen H, Myhrstad MC, et al: Polyphenols and glutathione synthesis regulation. Am J Clin Nutr 2005, 81:277S-283S.

41. Percival M: Phytonutrients and detoxification. Clinical Nutrition Insights 1997, 5:1-4.

42. Raucy JL: Regulation of CYP3A4 expression in human hepatocytes by pharmaceuticals and natural products. Drug Metab Dispos 2003, 31:533-539.

43. Nelson DR: Cytochrome P450 and the individuality of species. Arch Biochem Biophys 1999, 369:1-10.

44. Brigelius-Flohe R: Vitamin E and drug metabolism. Biochem Biophys Res Commun 2003, 305:737-740.

45. D'Angelo S, Manna C, Migliardi V, et al: Pharmacokinetics and metabolism of hydroxytyrosol, a natural antioxidant from olive oil. Drug Metab Dispos 2001, 29:1492-1498.

46. Mei N, Guo L, Zhang $L$, et al: Analysis of gene expression changes in relation to toxicity and tumorigenesis in the livers of Big Blue transgenic rats fed comfrey (Symphytum officinale). BMC Bioinformatics 2006, 7(Suppl 2):S16.

47. Nakashima $Y$, Ono T, Yamanoi A, et al: Expression of gap junction protein connexin32 in chronic hepatitis, liver cirrhosis, and hepatocellular carcinoma. J Gastroenterol 2004, 39:763-768.

48. Guo L, Mei N, Dial S, et al: Comparison of gene expression profiles altered by comfrey and riddelliine in rat liver. BMC Bioinformatics 2007, 8(Suppl 7):S22.

49. Maxwell KN, Soccio RE, Duncan EM, et al: Novel putative SREBP and LXR target genes identified by microarray analysis in liver of cholesterol-fed mice. J Lipid Res 2003, 44:2109-2119.

50. Breton AM: The spleen: Anatomy and common complications. Veterinary Technician 2005, 26:554-564.

51. Strom CC, Aplin M, Ploug T, et al: Expression profiling reveals differences in metabolic gene expression between exercise-induced cardiac effects and maladaptive cardiac hypertrophy. Febs J 2005, 272:2684-2695.

52. Fisler JS, Warden $\mathrm{CH}$ : Uncoupling proteins, dietary fat and the metabolic syndrome. Nutr Metab (Lond) 2006, 3:38.

53. Lee CK, Allison DB, Brand J, et al: Transcriptional profiles associated with aging and middle age-onset caloric restriction in mouse hearts. Proc Natl Acad Sci USA 2002, 99:14988-14993.

54. Dhahbi JM, Tsuchiya T, Kim HJ, et al: Gene expression and physiologic responses of the heart to the initiation and withdrawal of caloric restriction. J Gerontol A Biol Sci Med Sci 2006, 61:218-231.

55. Hansen JM, Watson WH, Jones DP: Compartmentation of Nrf-2 redox control: Regulation of cytoplasmic activation by glutathione and DNA binding by thioredoxin-1. Toxicol Sci 2004, 82:308-317.

56. Huang $Y$, Yan J, Lubet $R$, et al: Identification of novel transcriptional networks in response to treatment with the anticarcinogen $3 \mathrm{H}-1,2$ dithiole-3-thione. Physiol Genomics 2006, 24:144-153.

57. Matsui N, Ito R, Nishimura E, et al: Ingested cocoa can prevent high-fat diet-induced obesity by regulating the expression of genes for fatty acid metabolism. Nutrition 2005, 21:594-601.

58. de Wit NJ, Bosch-Vermeulen H, de Groot PJ, et al: The role of the small intestine in the development of dietary fat-induced obesity and insulin resistance in C57BL/6J mice. BMC Med Genomics 2008, 1:14.

59. Edgar R, Domrachev M, Lash AE: Gene Expression Omnibus: NCBI gene expression and hybridization array data repository. Nucleic Acids Res 2002, 30:207-210.

60. Saeed Al, Sharov V, White J, et al: TM4: A free, open-source system for microarray data management and analysis. Biotechniques 2003, 34:374-378.

61. Novak JP, Miller MC, Bell DA: Variation in fiberoptic bead-based oligonucleotide microarrays: Dispersion characteristics among hybridization and biological replicate samples. Biol Direct 2006, 1:18.

62. Bustin SA, Nolan T: Pitfalls of quantitative real-time reverse-transcription polymerase chain reaction. J Biomol Tech 2004, 15:155-166.

63. Nolan T, Hands RE, Bustin SA: Quantification of mRNA using real-time RTPCR. Nat Protoc 2006, 1:1559-1582.

64. Hellemans J, Mortier G, De Paepe A, et al: qBase relative quantification framework and software for management and automated analysis of real-time quantitative PCR data. Genome Biol 2007, 8:R19.

65. Vandesompele J, De Preter K, Pattyn F, et al: Accurate normalization of real-time quantitative RT-PCR data by geometric averaging of multiple internal control genes. Genome Biol 2002, 3:RESEARCH0034. doi:10.1186/1471-2164-12-432

Cite this article as: Leow et al.: Differential transcriptomic profiles effected by oil palm phenolics indicate novel health outcomes. BMC Genomics 2011 12:432.

\section{Submit your next manuscript to BioMed Central and take full advantage of:}

- Convenient online submission

- Thorough peer review

- No space constraints or color figure charges

- Immediate publication on acceptance

- Inclusion in PubMed, CAS, Scopus and Google Scholar

- Research which is freely available for redistribution

Submit your manuscript at www.biomedcentral.com/submit
Biomed Central 